\title{
Feedback Based Bandwidth Allocation with Call Admission Control for Providing Delay Guarantees in IEEE 802.11e Networks *
}

\author{
G. Boggia, P. Camarda, L. A. Grieco, S. Mascolo* \\ DEE, Politecnico di Bari, Via Orabona 4, 70125 BARI, Italy \\ \{g.boggia;camarda;a.grieco;mascolo\}@poliba.it
}

\begin{abstract}
The 802.11 e working group has recently proposed innovative functionalities in order to support delay-sensitive multimedia applications, such as real-time voice and video, in Wireless Local Area Networks (WLANs). In particular, the 802.11e proposal introduces: (1) the Hybrid Coordination Function (HCF), which is an improved channel access method aimed at allocating the first-hop WLAN bandwidth to delay-insensitive and delay-sensitive flows; (2) a Call Admission Control (CAC) algorithm for preventing network overloads, which would drastically degrade the service offered by the network; (3) and a Signalling scheme for service request and QoS service level negotiation.

This paper proposes a dynamic bandwidth allocation algorithm along with a measurement based CAC algorithm for providing delay guarantees to real-time media flows in IEEE 802.11e networks. The dynamic bandwidth allocation algorithm has been designed using classic feedback control; whereas the CAC scheme is an extension of the one proposed by the $802.11 \mathrm{e}$ working group because it takes into account the actually used resources rather than the average rates declared by data sources. Both of the schemes exploit the HCF Controlled Channel Access (HCCA) centralized access method.

The proposed algorithms have been implemented in the $n s-2$ simulator and extensive computer simulations have been carried out to assess their validity. Simulation results have shown that proposed algorithms protect the WLAN from heavy overloads and guarantees bounded delays for multimedia flows, whereas, analogous algorithms proposed by the 802.11 e working group fail when in the presence of high network loads.
\end{abstract}

Key words: Quality of Service, Wireless LAN, Dynamic Bandwidth Allocation, Feedback Control 


\section{Introduction}

The widespread deployment of IEEE 802.11 Wireless Local Area Networks (WLANs) as a fundamental tool for enabling ubiquitous wireless networking is mainly due to their easy installation, flexibility and robustness against failures $[1,2]$. The $802.11 \mathrm{a}$ and $802.11 \mathrm{~g}$ versions allow a data rate up to $54 \mathrm{Mbps}$, using Orthogonal Frequency Division Multiplexing technique in the unlicensed $5 \mathrm{GHz}$ band and in the ISM $2.4 \mathrm{GHz}$ band, respectively [3,4]. The 802 High Throughput Task Group, known as $802.11 n$, is exploring ways to increase bit rates up to $108 \mathrm{Mbps}$ and, possibly by 2005, up to 320 Mbps [2].

The 802.11 Medium Access Control (MAC) employs a mandatory contentionbased channel access scheme called Distributed Coordination Function (DCF), which is based on Carrier Sense Multiple Access with Collision Avoidance (CSMA/CA) [5] and an optional centrally controlled channel access scheme called Point Coordination Function (PCF) [1].

The fundamental building block of the IEEE 802.11 architecture is the Basic Service Set (BSS), which is composed by a group of stations (STAs), located in the same geographical area, that access the radio channel under the direct control of a single coordination function(i. e., DCF or PCF) [1,5-7]. The 802.11 standard allows two topological configurations that are the Independent Basic Service Set (IBSS) and the Extended Service Set (ESS) [1,5]. An IBSS is a particular BSS where any station can establish a direct communication session with any other station in the IBSS (i.e., an ad hoc network architecture). An ESS, instead, is composed by one or more interconnected BSS, which are linked to each other via a common distribution system, generally wired. In the case of ESS, stations within each BSS cannot communicate to each other directly but the traffic is channeled through a central station, which is referred to as Access Point (AP) and is responsible also for inter-BSS communications [1,5]. In the sequel we will always assume ESS configurations.

At present, the use of WLANs has been essentially focused on best effort data transfer because the basic DCF and PCF access methods cannot provide delay guarantees to real-time multimedia flows [8-10]. Recently, in order to support also delay-sensitive multimedia applications, such as real-time voice and video, the $802.11 \mathrm{e}$ working group has proposed innovative functionalities, which enable service differentiation in WLANs. In particular, the 802.11e proposal introduces: (1) the Hybrid Coordination Function (HCF), which is an enhanced access method to distribute the limited first-hop WLAN bandwidth among delay-insensitive and delay-sensitive flows; (2) a Call Admis-

\footnotetext{
* This is an extended version of the paper "A Dynamic Bandwidth Allocation Algorithm for IEEE 802.11e WLANs with HCF Access Method", Qofis 2003 workshop.

* Corresponding author
} 
sion Control (CAC) algorithm for preventing network overloads, which would drastically degrade the service offered by the network; (3) specific signalling messages for service request and Quality of Service (QoS) service level negotiation. The $802.11 \mathrm{e}$ draft does not specify an effective bandwidth allocation algorithm for providing the QoS required by real-time flows; it only suggests a simple scheduler that provides a Constant Bit Rate (CBR) service. This scheduler does not exploit any feedback information from mobile stations in order to dynamically assign the WLAN bandwidth. Thus, it is not well suited for bursty media flows [11]. An improved bandwidth allocation algorithm has been proposed in [12], which schedules transmission opportunities by taking into account both the average and the maximum source rates declared by each data source. However, also this scheme does not consider the dynamic behavior of multimedia flows. An adaptive version of the simple scheduler, which is based on the Delay-Earliest Due-Date algorithm, has been proposed in [11]. This algorithm implements a trial and error procedure to discover the optimal bandwidth assignment to each station.

This paper proposes a dynamic bandwidth allocation algorithm along with an associated CAC algorithm for providing delay guarantees to real-time media flows in IEEE 802.11e networks. The dynamic bandwidth allocation algorithm has been designed using classic feedback control theory [13-16] whereas the CAC scheme is an extension of the admission control algorithm proposed by the 802.11e working group. Both of them exploit the HCF Controlled Channel Access (HCCA) centralized access method. The developed schemes have been implemented in the $n s-2$ simulator and extensive computer simulations have been carried out to assess their performance. Simulation results have shown that the proposed algorithms are able to protect the WLAN from heavy overloads and to provide bounded delays to multimedia flows, whereas, the standard algorithms proposed by the 802.11e working group fail in the presence of high network loads.

The rest of the paper is organized as follows: Section 2 describes main functionalities of the 802.11 standard and the 802.11e QoS enhancements; Section 3 proposes and analyzes the dynamic bandwidth allocation algorithm, and illustrates the proposed CAC scheme; Section 4 shows simulation results; finally the last Section draws the conclusions.

\section{Overview of the IEEE 802.11 MAC}

The basic 802.11 MAC protocol is the Distributed Coordination Function (DCF), which is based on Carrier Sense Multiple Access/Collision Avoidance (CSMA/CA) mechanism: for each frame a station listens the channel before transmitting; if the station detects an idle channel for a minimum interval 
time called DCF Interframe Space (DIFS), then it transmits immediately a MAC Protocol Data Unit (MPDU). Otherwise, if the medium is sensed busy, transmission is deferred until the channel is sensed idle for a DIFS period plus an additional random backoff time. The backoff time is a multiple of a slot time, where the slot time depends on the physical layer implementation, and the multiple is an integer taken from a uniform distribution in the interval from 0 to the Contention Window (CW) [1].

Each correctly received frame is acknowledged by an ACK frame, that is sent after a Short Interframe Space (SIFS) period, which is shorter than a DIFS, to avoid that other stations use the channel. If the transmission is not successful, i.e., no ACK frame is received by the sending station, the listen before talking protocol and the backoff procedure are repeated by doubling the $\mathrm{CW}$ value up to the maximum limit of 1023 time slots.

The Collision Avoidance is obtained by Virtual Carrier Sensing: in the header of each delivered frame there is a duration field, which indicates the time required for transmission. The duration field is used by each station in the BSS to update its Network Allocation Vector (NAV), which accounts for the duration of the current transmission after which the channel can be sensed again for the access $[1,5]$.

In order to support time-sensitive services, the 802.11 standard defines also the Point Coordination Function (PCF) as an optional access method which provides a contention-free medium access. The Point Coordinator (PC), typically the AP within each BSS, polls the stations asking for time-sensitive service in a round robin fashion, and allows them to transmit a data frame without channel contention. With PCF, the time is divided into repeated periods, called SuperFrames (SFs), which consist of a Contention Period (CP) and a Contention Free Period (CFP). During the CP, the channel is accessed using the DCF whereas, during the CFP, is accessed using the PCF. Each superframe must contain a $\mathrm{CP}$ long enough to transmit at least one MPDU. The PC cannot dynamically allocate the wireless channel capacity by taking into account the status of mobile stations, because it does not know the starting time and the transmission duration of the polled stations under its coordination [8].

\subsection{IEEE 802.11e QoS enhancements}

In order to obtain service differentiation in 802.11 WLANs, the 802.11e working group has introduced new functionalities for QoS service level negotiation, Call Admission Control, and an improved access method, which is the Hybrid Coordination Function (HCF). 
Stations operating under 802.11e specifications are usually known as enhanced stations or QoS Stations (QSTAs). 802.11e defines 8 Traffic Categories (TCs) with the priority values of the IEEE 802.1D standard [17]. TCs are characterized by traffic specifications (TSPECs) similar to those introduced in [18] for IP FlowSPecs definition and adopted in IntServ [19] and DiffServ [20] architectures. Four Access Categories (ACs) have been introduced in order to support the mentioned eight TCs (the mapping between TCs and ACs is shown in Table 1) [21]. To satisfy the QoS requirements of each AC, the concept of TXOP (Transmission Opportunity) is introduced, which is defined as the time interval during which a station has the right to transmit. The contiguous time during which TXOPs are granted to the same QSTA is called Service Period (SP). The interval $T_{S I}$ between two successive SPs is called Service Interval $[8,21]$.

Table 1

Mapping Between Traffic and Access Categories

\begin{tabular}{ccc}
\hline Traffic Category & Access Category & Designation \\
\hline \hline 1 & AC_BK & Background \\
2 & AC_BK & Background \\
0 & AC_BE & Best Effort \\
3 & AC_BE & Best Effort \\
4 & AC_VI & Video \\
5 & AC_VI & Video \\
6 & AC_VO & Voice \\
7 & AC_VO & Voice \\
\hline
\end{tabular}

\subsubsection{The Hybrid Coordination Function (HCF)}

The HCF is made of a contention-based channel access, known as the Enhanced Distributed Coordination Access (EDCA), and of a HCF Controlled Channel Access (HCCA). The use of the HCF requires a centralized controller, which is called the Hybrid Coordinator (HC) and is generally located at the access point.

The EDCA method operates as the basic DCF access method but using different contention parameters per access category. In this way, a service differentiation among ACs is statistically pursued [22]. A queue is associated to each AC at any QSTA, which acts as a virtual station with its own QoS parameters. Each queue within a station contends for a TXOP and starts a backoff timer after detecting that the channel is idle for an Arbitration Interframe Space (AIFS), which is at least equal to a DIFS. For each class $\mathrm{AC}(i)$, a contention window $\mathrm{CW}(i)$ and an $\operatorname{AIFS}(i)$ are defined as shown in 
Table 2 [23]. If several backoff timers reach zero within the same station at the same time slot, then the highest priority frame will be transmitted and any lower priority frame will be deferred with the retry procedure and modifying the backoff timer $[8,21]$.

EDCA parameters have to be properly set to provide prioritization of ACs. Tuning them in order to meet specific QoS needs is a current research topic. A method to set these parameters to guarantee throughput of each TC has been described in [24]. Regarding the goal of providing delay guarantees, several papers have pointed out that the EDCA behavior is very sensitive to the value of the contention parameters $[23,25]$ and that the Interframe-space-based priority scheme used by the EDCA mechanism can provide only a relative differentiation among service classes, but not absolute guarantees on throughput/delay performance [22]. Finally, in the paper [26] it has been shown that that EDCA can starve lower priority flows. To overcome these limitations, adaptive algorithms that dynamically tune EDCA parameters have been recently proposed in $[27,28]$, however, the effectiveness of these heuristic schemes have been proved only using simulations and no theoretical bounds on their performance in a general scenario has been derived.

Table 2

TYPICAL VALUES OF EDCA CONTENTION PARAMETERS

\begin{tabular}{cccc}
\hline $\mathrm{AC}$ & $C W_{\min }$ & $C W_{\max }$ & AIFS \\
\hline \hline $\mathrm{AC} \_\mathrm{BK}$ & $C W_{\min }$ & $C W_{\max }$ & 7 \\
$\mathrm{AC} \_\mathrm{BE}$ & $C W_{\min }$ & $C W_{\max }$ & 3 \\
$\mathrm{AC} \_V I$ & $\left(C W_{\min }+1\right) / 2-1$ & $C W_{\min }$ & 2 \\
$\mathrm{AC} \_V O$ & $\left(C W_{\min }+1\right) / 4-1$ & $\left(C W_{\min }+1\right) / 2-1$ & 2 \\
\hline
\end{tabular}

The HCCA method combines some of the EDCA characteristics with some of the PCF basic features as it is shown in Fig. 1. Each superframe starts with a beacon frame after which, for legacy purpose, there could be a contention free period for PCF access. The remaining part of the superframe forms the $\mathrm{CP}$, during which the QSTAs contend to access the radio channel using the EDCA mechanism.

After the medium remains idle for at least a PIFS interval during the $\mathrm{CP}$, the $\mathrm{HC}$ can start a Contention Access Phase $(\mathrm{CAP})^{1}$. During the CAP, only QSTAs polled and granted with a special frame, known as QoS CF-Poll frame, are allowed to transmit during their TXOPs. Thus, the HC implements a prioritized medium access control. Notice that PIFS is shorter than each AIFS. The number of CAPs and their locations in each superframe are chosen by

$\overline{1 \text { HCCA }}$ can be also enabled during the CFP with a procedure similar to the one described in this Section. 


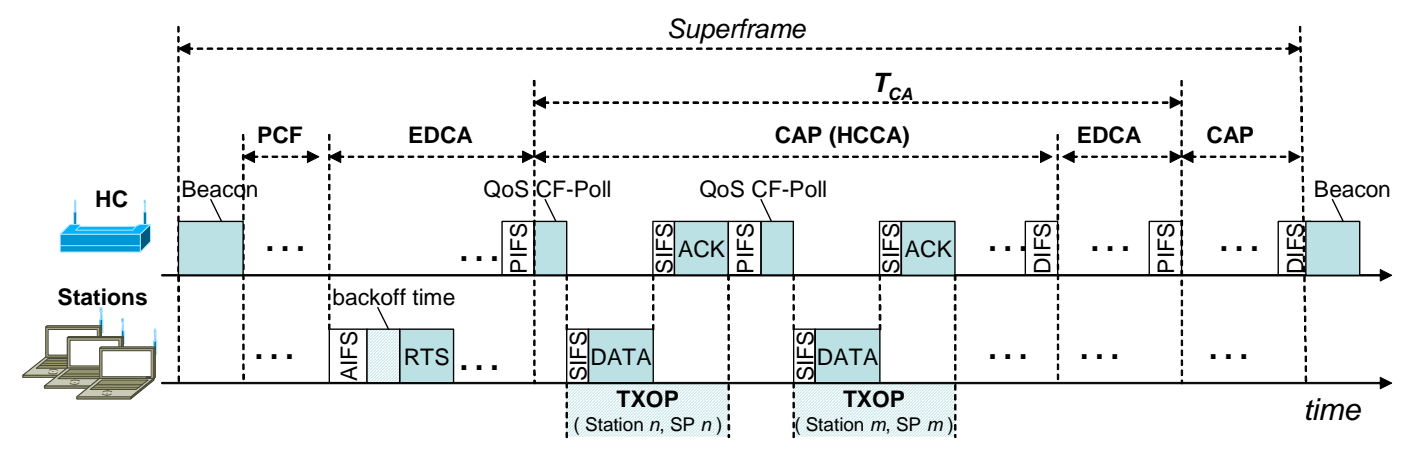

Fig. 1. Scheme of a superframe using the HCF controlled access method.

the $\mathrm{HC}$ in order to satisfy QoS needs of each station. Moreover, at least one $\mathrm{CP}$ interval, long enough to transmit a maximum size data frame at the minimum BSS rate, must be contained in a superframe; this CP interval can be used for management tasks, such as associations of new stations, new traffic negotiations, and so on. CAP length cannot exceed the value of the system variable dot11CAPLimit, which is advertised by the $\mathrm{HC}$ in the Beacon frame when each superframe starts [21].

The IEEE 802.11e specifications allow QSTAs to feed back queue lengths of each AC to the HC. This information is carried on a 8 bits long subfield contained in the QoS Control Field of each frame header, during data transmission both in the CAPs and in the CPs; queue lengths are reported in units of 256 octets. This information can be used to design novel HCCA-based dynamic bandwidth allocation algorithms using feedback control [14,15], as will be shown in this paper. In fact, the 802.11e draft does not specify how to schedule TXOPs in order to provide the required QoS; it only suggests a simple scheduler that uses static values declared in TSPECs for assigning fixed TXOPs (more details about this scheduler can be found in [21] and [11]). In particular, the simple scheduler designed in the draft [21] states that the $T X O P_{i}$ assigned to the $i^{\text {th }}$ queue should be computed as follows:

$$
T X O P_{i}=\max \left(\frac{N_{i} \cdot L_{i}}{C_{i}}+O, \frac{M}{C_{i}}+O\right)
$$

where $L_{i}$ is the nominal MAC Service Data Unit (MSDU) size associated with the $i^{\text {th }}$ queue; $C_{i}$ is the physical data rate at which the data of the $i^{\text {th }}$ queue are transmitted over the WLAN; $O$ is the overhead due to ACK packets, SIFS and PIFS time intervals; $M$ is the maximum MSDU size; and $N_{i}=\left\lceil\frac{T_{S I} \cdot \rho_{i}}{L_{i}}\right\rceil$, where $\rho_{i}$ is the Mean Data Rate associated with the $i^{t h}$ queue and $T_{S I}$ is the Service Interval. This scheduler does not exploit any feedback information from mobile stations in order to dynamically assign TXOPs. Thus, it is not well suited for bursty media flows [11]. An improved bandwidth allocation algorithm has been proposed in [12], which schedules transmission opportunities by taking into account both the average and the maximum source rates declared in the 
TSPECs. However also this scheme does not consider the dynamic behavior of the multimedia flows. An adaptive version of the simple scheduler, which is based on the Delay-Earliest Due-Date algorithm, has been proposed in [11]. However, this algorithm does not exploit the explicit queue length to assign TXOPs, but implements a trial and error procedure to discover the optimal TXOP to be assigned to each station.

In the following section, we will design a novel closed-loop control scheme based on feeding back queue levels to guarantee the typical QoS requirements of real-time audio/video applications.

\subsubsection{QoS signalling}

Service request and Service level negotiation are new functionalities introduced by the 802.11e proposal [21] in order to support QoS in 802.11 networks. Each Traffic Stream (TS), i.e., a data flow with QoS needs, is described by a Traffic SPECification (TSPEC), which indicates the main characteristics of the TS (see Table 3) [21].

When a new TS has to be started, the following steps are executed considering that each node has a MAC layer and a Management Entity (see also the Message Sequence Chart in Fig. 2):

(1) The Station Management Entity (SME) of the QSTA with the new TS request issues a setup phase by generating a message, which is known as Mac Layer Management Entity (MLME)-ADDTS request and contains the TSPEC of the TS.

(2) The QSTA MAC layer transmits this request to the $\mathrm{HC}$ and starts a response timer (known as ADDTS timer), the duration of the ADDTS Timer is defined by the system variable dot11ADDTSResponseTimeout.

(3) After receiving the request, the MAC layer of the HC generates the message MLME-ADDTS indication for its SME which contains the TSPEC.

(4) The SME in the HC decides whether to admit the TS with the specified TSPEC, or to refuse it or to not admit it suggesting an alternative TSPEC.

(5) The HC MAC transmits the ADDTS response.

(6) The QSTA MAC layer receives this management frame and reset the ADDTS timer. Then it sends a MLME-ADDTS confirm message to its SME. The QSTA SME decides whether the response meets its needs or not. If the ResultCode is "SUCCESS", the TS enters into an active state. Otherwise, the whole process can be repeated [1]. 
Table 3

Some TSPEC PARAmeters

\begin{tabular}{|c|c|}
\hline Parameter & Description \\
\hline$T S I D$ & Traffic Stream (TS) Identifier \\
\hline Direction & Direction of data carried by the TS \\
\hline $\begin{array}{l}\text { Nominal/Maximum } \quad M S D U \\
\text { sizes }\end{array}$ & Nominal/Maximum sizes of MAC frames \\
\hline $\begin{array}{l}\text { Minimum/Mean/Peak Data } \\
\text { Rate }\end{array}$ & Characteristics of source data rate \\
\hline $\begin{array}{l}\text { Minimum/Maximum Service } \\
\text { Interval }\end{array}$ & $\begin{array}{l}\text { Minimum/maximum interval between two con- } \\
\text { secutive service periods }\end{array}$ \\
\hline Maximum Burst Size & $\begin{array}{l}\text { Maximum burst size, in octets, of the MSDUs } \\
\text { belonging to TS that arrive at the peak data } \\
\text { rate }\end{array}$ \\
\hline Minimum PHY Rate & Desired minimum PHY rate to use for the TS \\
\hline Surplus Bandwidth Allowance & $\begin{array}{l}\text { Surplus of bandwidth allowed for a correct } \\
\text { transmission of the MSDUs, taking into account } \\
\text { retransmissions }\end{array}$ \\
\hline Delay Bound & $\begin{array}{l}\text { Maximum amount of time allowed to transport } \\
\text { an MSDU belonging to the TS }\end{array}$ \\
\hline Inactivity Interval & $\begin{array}{l}\text { Maximum amount of time (in microseconds) } \\
\text { that may elapse without arrival or transfer of } \\
\text { an MSDU belonging to the TS before this TS is } \\
\text { deleted by the MAC entity at the HC }\end{array}$ \\
\hline
\end{tabular}

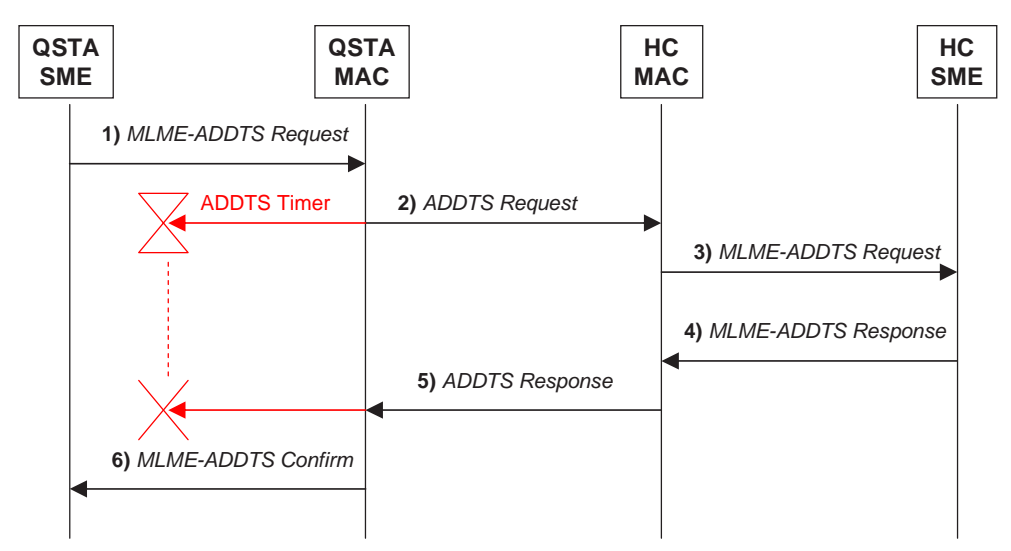

Fig. 2. Message Sequence Chart of Traffic Stream setup.

\subsubsection{Call Admission Control}

An IEEE 802.11 network may use admission control to administer the available bandwidth resources. The $\mathrm{HC}$ is used as admission control unit. Since the QoS facility supports two access mechanisms, there are two distinct ad- 
mission control mechanisms: one for contention-based access and the other for controlled-access. Herein, we will focus on the latter mechanism since the algorithms proposed in this paper are based on the controlled access mechanism HCCA. Details regarding the contention-based admission control can be found in [21].

Byu assuming that $k$ is the number of admitted flows, when a new TS requests admission, the Admission Control Unit (ACU), calculates the TXOP duration that needs to be allocated for the stream $\left(T X O P_{k+1}\right)$ accordingly to Eq. (1). The stream is admitted if the following inequality is satisfied:

$$
\frac{T X O P_{k+1}}{T_{S I}}+\sum_{i=1}^{k} \frac{T X O P_{i}}{T_{S I}} \leq \frac{T-T_{C P}}{T}
$$

where $T$ indicates the superframe duration and $T_{C P}$ is the time used for EDCA traffic during the superframe.

\section{HCCA-based Dynamic Bandwidth Allocation}

This section proposes a HCCA-based bandwidth allocation algorithm. The algorithm, which has been designed using classical feedback control theory, distributes the WLAN bandwidth among all the multimedia flows by taking into account the queue levels fed back by the QSTAs [29]. Bandwidth allocation is pursued by exploiting the HCCA functionalities, which allows the $\mathrm{HC}$ to assign TXOPs to the ACs by taking into account the specific time constraints of each AC.

We will refer to a WLAN system made of an Access Point (AP) and a set of quality of service enabled mobile stations (QSTAs). Each QSTA has $N$ queues, with $N \leq 4$, one for any AC in the 802.11e proposal. Let $T_{C A}$ be the time interval between two successive CAPs. Every time interval $T_{C A}$, assumed constant, the AP must allocate the bandwidth that will drain each queue during the next CAP. We assume that at the beginning of each CAP, the $\mathrm{AP}$ is aware of all the queue levels $q_{i}, i=1, \ldots, M$ at the beginning of the previous CAP, where $M$ is the total number of traffic queues in the WLAN system. The latter is a worst case assumption, in fact, queue levels are fed back using frame headers as described in Sec. 2.1; as a consequence, if the $i^{\text {th }}$ queue length has been fed at the beginning of the previous CAP, then the feedback signal might be delayed up to $T_{C A}$ seconds.

The dynamics of the $i^{t h}$ queue can be described by the following discrete time 
linear model:

$$
q_{i}(k+1)=q_{i}(k)+d_{i}(k) \cdot T_{C A}+u_{i}(k) \cdot T_{C A}, \quad i=1, \ldots, M,
$$

where $q_{i}(k) \geq 0$ is the $i^{t h}$ queue level at the beginning of the $k^{t h} \mathrm{CAP} ; u_{i}(k) \leq 0$ is the average depletion rate of the $i^{\text {th }}$ queue (i.e., the bandwidth assigned to drain the $i^{t h}$ queue); $d_{i}(k)=d_{i}^{s}(k)-d_{i}^{C P}(k)$ is the difference between $d_{i}^{s}(k) \geq 0$, which is the average input rate at the $i^{\text {th }}$ queue during the $k^{\text {th }} \mathrm{CAP}$, and $d_{i}^{C P}(k) \geq 0$, which is the amount of data transmitted by the $i^{\text {th }}$ queue during the $k^{\text {th }}$ CAP divided by $T_{C A}$.

The signal $d_{i}(k)$ is unpredictable since it depends on the behavior of the source that feeds the $i^{\text {th }}$ queue and on the number of packets transmitted during the contention periods. Thus, from a control theoretic perspective, $d_{i}(k)$ can be modelled as a disturbance. Without loss of generality, the following piece-wise constant model for the disturbance $d_{i}(k)$ can be assumed [14]:

$$
d_{i}(k)=\sum_{j=0}^{+\infty} d_{0 j} \cdot 1\left(k-t_{j}\right)
$$

where $1(k)$ is the unitary step function, $d_{0 j} \in \mathbb{R}$, and $t_{j}$ is a time lag.

Due to the assumption (4), the linearity of the system (3), and the superposition principle that holds for linear systems, we will design the feedback control law by considering only a step disturbance: $d_{i}(k)=d_{0} \cdot 1(k)$.

\subsection{Control law and stability analysis}

Our goal is to design a control law that drives the queuing delay $\tau_{i}$ experienced by each frame going through the $i^{\text {th }}$ queue to a desired target value $\tau_{i}^{T}$ that represents the QoS requirement of the $\mathrm{AC}$ associated to the queue.

For the time being, we start by setting a constant reference length $q_{i}^{T}$ for each queue $q_{i}$ and we design a closed loop control algorithm aiming at driving each queue level to its target. Then we will show how this scheme can be used for our purpose.

In particular, we consider the closed-loop control scheme depicted in Fig. 3, which gives the way to compute the $\mathcal{Z}$-transform of $q_{i}(k)$ and $u_{i}(k)$ as follows:

$$
\begin{aligned}
& Q_{i}(z)=\frac{z \cdot k_{i} \cdot T_{C A}}{z^{2}-z+k_{i} \cdot T_{C A}} \cdot Q_{i}^{T}(z)+\frac{z \cdot T_{C A}}{z^{2}-z+k_{i} \cdot T_{C A}} \cdot D_{i}(z) \\
& U_{i}(z)=\frac{z \cdot k_{i} \cdot(z-1)}{z^{2}-z+k_{i} \cdot T_{C A}} \cdot Q_{i}^{T}(z)-\frac{k_{i} \cdot T_{C A}}{z^{2}-z+k_{i} \cdot T_{C A}} \cdot D_{i}(z)
\end{aligned}
$$


whit $D_{i}(z)=\mathcal{Z}\left[d_{i}(k)\right]$ and $Q_{i}^{T}(z)=\mathcal{Z}\left[q_{i}^{T}\right]$.

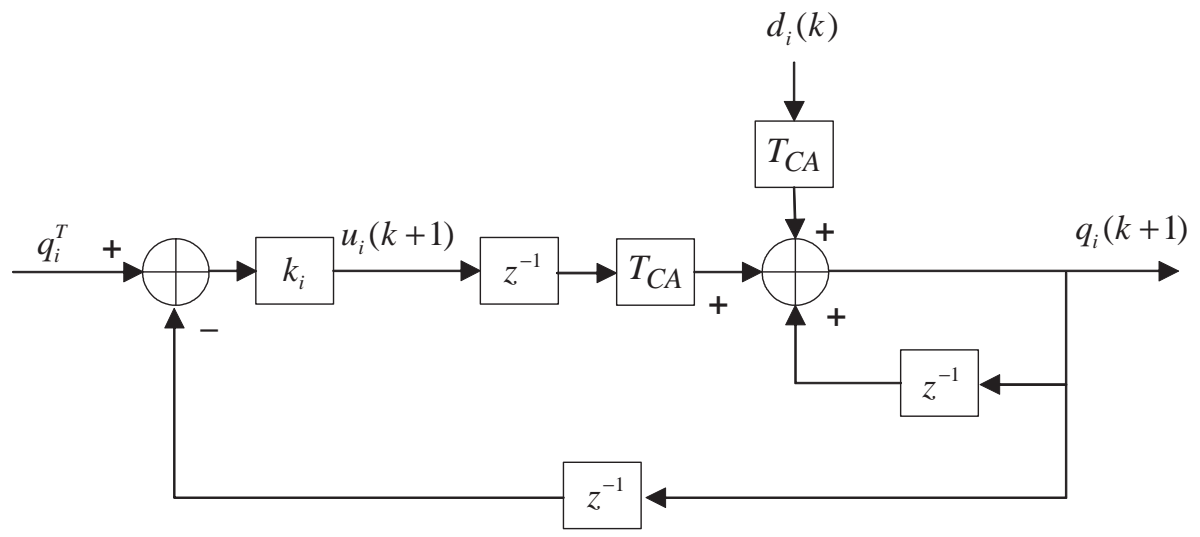

Fig. 3. HCCA-based closed-loop control scheme.

From Eqs. (5) and (6) the system poles are $z_{p}=\frac{1 \pm \sqrt{1-4 k_{i} \cdot T_{C A}}}{2}$, which give an asymptotically stable system if and only if $\left|z_{p}\right|<1$, that is:

$$
0<k_{i}<\frac{1}{T_{C A}}
$$

In the sequel, we will always assume that $k_{i}$ satisfies this asymptotic stability condition stated by (7).

To investigate the ability of the control system to provide a queuing delays approaching the target value $\tau_{i}^{T}$, we apply the final value theorem to Eqs. (5) and (6). By considering that the $\mathcal{Z}$-transforms of the step functions $q_{i}^{T}(k)=$ $q_{i}^{T} \cdot 1(k)$ and $d_{i}(k)=d_{0} \cdot 1(k)$ are $Q_{i}^{T}(z)=q_{i}^{T} \cdot \frac{z}{z-1}$ and $D_{i}(z)=d_{0} \cdot \frac{z}{z-1}$, respectively, the following results turn out:

$$
\begin{gathered}
u_{i}(+\infty)=\lim _{k \rightarrow+\infty} u_{i}(k)=\lim _{z \rightarrow 1}(z-1) U_{i}(z)=-d_{0} ; \\
q_{i}(+\infty)=q_{i}^{T}+\frac{d_{0}}{k_{i}},
\end{gathered}
$$

which implies that the the steady state queueing delay is:

$$
\tau_{i}(+\infty)=\left|\frac{q_{i}(+\infty)}{u_{i}(+\infty)}\right|=\frac{q_{i}^{T}}{d_{0}}+\frac{1}{k_{i}} .
$$

Setting $q_{i}^{T}=0$, which implies that we would ideally obtain empty queues, the following inequality has to be satisfied in order to achieve a steady-state delay smaller than $\tau_{i}^{T}$ :

$$
k_{i} \geq \frac{1}{\tau_{i}^{T}}
$$


By considering inequalities (7) and (9) we obtain that the $T_{C A}$ parameter has to fulfill the following constraint:

$$
T_{C A}<\min _{i=1 . . M} \tau_{i}^{T}
$$

\subsection{TXOP assignment}

We have seen in Sec. 2.1 that every time interval $T_{C A}$ the HC allocates TXOPs to mobile stations in order to meet the QoS constraints. This sub-section shows how to transform the bandwidth $u_{i}$ into a $T X O P_{i}$ assignment. In particular, if the $i^{t h}$ queue is drained at data rate $C_{i}$, the following relation holds:

$$
T X O P_{i}(k)=\frac{\left|u_{i}(k) \cdot T_{C A}\right|}{C_{i}}+O
$$

where $\operatorname{TXOP}(k)$ is the TXOP assigned to the $i^{\text {th }}$ queue during the $k^{\text {th }}$ service interval and $O$ is the time overhead due to ACK packets, SIFS and PIFS time intervals (see Fig. 1). The extra quota of TXOP due to the overhead $O$ depends on the number of MSDUs corresponding to the amount of data $\left|u_{i}(k) \cdot T_{C A}\right|$ to be transmitted. $O$ could be estimated by assuming that all MSDUs have the same nominal size specified into the TSPEC. Moreover, when $\left|u_{i}(k) \cdot T_{C A}\right|$ does not correspond to a multiple of MSDUs, the TXOP assignment will be rounded in excess in order guarantee a queuing delay always equal or smaller than the target delay $\tau_{i}^{T}$.

\subsection{Channel saturation}

The above bandwidth allocation algorithm is based on the implicit assumption that the sum of the TXOPs assigned to each queue is smaller than the maximum CAP duration, which is the dot11CAPLimit; this value can be violated when the network is saturated. In order to avoid channel saturations, we will adopt a CAC scheme, which will be described in the next Section and has been obtained by improving the one proposed by the $802.11 \mathrm{e}$ working group. However, since transient network overloads cannot be avoided due to the burstiness of the multimedia flows, it is necessary to reallocate the TXOPs to avoid exceeding the CAP limit. This task is performed as follows: when $\sum_{i=1}^{M} T X O P_{i}\left(k_{0}\right)>\operatorname{dot} 11 C A P$ Limit, each computed $T X O P_{i}\left(k_{0}\right)$ is decreased by an amount $\triangle T X O P_{i}\left(k_{0}\right)$, so that the capacity constraints

$$
\sum_{i=1}^{M}\left[T X O P_{i}\left(k_{0}\right)-\Delta T X O P_{i}\left(k_{0}\right)\right]=\operatorname{dot} 11 C \text { APLimit }
$$


is satisfied.

In particular, the generic amount $\triangle T X O P_{i}\left(k_{0}\right)$ is evaluated as a fraction of the total amount $\sum_{i=1}^{M} T X O P_{i}\left(k_{0}\right)-\operatorname{dot} 11 C A P L i m i t$, as follows:

$$
\Delta T X O P_{i}\left(k_{0}\right)=\frac{T X O P_{i}\left(k_{0}\right) C_{i}}{\sum_{i=1}^{M}\left[T X O P_{i}\left(k_{0}\right) C_{i}\right]}\left(\sum_{i=1}^{M} T X O P_{i}\left(k_{0}\right)-\operatorname{dot} 11 C A P \text { Limit }\right) .
$$

Notice that Eq. (13) provides a $\triangle T X O P_{i}\left(k_{0}\right)$, which is proportional to $T X O P_{i}\left(k_{0}\right) C_{i}$, in this way connections transmitting at low rates are not penalized too much.

\subsection{Call Admission Control}

When the number of multimedia flows sharing the WLAN increases and the channel saturates and delay bounds cannot be guaranteed [28]. Under these conditions, a Call Admission Control scheme is required in guarantee QoS. Herein we describe the proposed CAC scheme, which exploits the 802.11e CAC proposal (see Sec. 2.1).

In particular, starting from the TXOPs allocated to the active traffic streams in each CAP, a new flow request is admitted if the following inequality is satisfied:

$$
\frac{T X O P_{k+1}}{T_{C A}}+\sum_{i=1}^{k} \frac{T X O P_{i}}{T_{C A}} \leq \frac{T-T_{C P}}{T}
$$

where $T$ indicates the superframe duration and $T_{C P}$ is the time used for EDCA traffic during the superframe.

Notice that the proposed CAC test (14) has been obtained from the standard CAC test (2), by replacing the constant TXOPs used by the simple scheduler with the time-varying ones assigned by our bandwidth allocation algorithm. In this way, the $\mathrm{CAC}$ test takes into account the bandwidth actually used by the flows and not just the sum of the average source rates declared in the TSPECs.

\subsection{System Design}

Starting from the theoretical results obtained in the previous sub-sections, we provide guidelines to design a dynamic bandwidth allocation algorithm in the 802.11e using the HCF access method. In particular, we will show how to set 
the WLAN parameters to meet the QoS requirements of each traffic category.

Table 4 reports typical QoS specifications for audio/video applications [30]. Real time audio/video applications, such as teleconference, exhibit the more

Table 4

Examples of performance targets for audio and video applications

\begin{tabular}{|l|l|l|}
\hline Class Service & Target One Way Delay & $\begin{array}{l}\text { Target Delay } \\
\text { Variation }\end{array}$ \\
\hline Conversational voice & preferred: $<150 \mathrm{~ms}$, limit: $400 \mathrm{~ms}$ & $<1 \mathrm{~ms}$ \\
Videophone & preferred: $<150 \mathrm{~ms}$, limit: $400 \mathrm{~ms}$ & $<1 \mathrm{~ms}$ \\
One Way Video & $<10 \mathrm{~s}$ & N.A. \\
\hline
\end{tabular}

stringent QoS specifications. In fact, they require an end-to-end delay smaller than $150 \mathrm{~ms}$ and a jitter smaller than $1 \mathrm{~ms}$. By using adequate echo control, the one way delay can reach the value $400 \mathrm{~ms}$ [30]. Since the WLAN is only the first hop encountered by a media flow, it is necessary to guarantee a delay smaller than the end-to-end expected delay. For these considerations, we choose a target delay equal to $30 \mathrm{~ms}$, that is $\tau_{i}^{T}=30 \mathrm{~ms}$. In order to satisfy inequality (10), we must set $T_{C A} \leq \min _{i=1 . . M}\left(\tau_{i}^{T}\right)=30 \mathrm{~ms}$. From (7) it results that, in order to guarantee system stability the gain must satisfy the inequality $k_{i} \leq \frac{1}{T_{C A}}=33.3 \mathrm{~s}^{-1}$.

To give a further insight on the influence of $T_{C A}$ parameter on the system performance, it should be considered that, due to the granularity of the system, the transmission of a packet can be delayed up to $\tau_{i}^{T}+T_{C A}$. Thus, a large $T_{C A}$ would critically affect queuing delay of media flows carrying conversational voice or video.

\subsection{Computational Complexity}

Herein we estimate the computational complexity of the proposed bandwidth allocation algorithm. Channel saturation episodes will be neglected because we assume they are sporadic owing to the effectiveness of the admission control scheme. At the beginning of each beacon interval, the HC has to compute the bandwidth assignment for each one of the $M$ active traffic stream. Accordingly to Eq. (11), a single bandwidth assignment consists of two multiplications and one sum. The first multiplication takes into account the TXOP assignment, the second one estimates the overhead due to ACK packets, SIFS and PIFS. Thus, we need $2 M$ multiplication plus a sum for each superframe. Therefore, the algorithm complexity scales linearly with the size of the system. Such 
a worth feature is mainly due to the simplicity of the adopted proportional feedback controller.

\section{Performance Evaluation}

In order to test the proposed bandwidth allocation algorithm in realistic scenarios, computer simulations involving voice, video and FTP data transfers have been run using the $n s-2$ simulator [31]. We have considered the reference scenario shown in Fig. 4 where a 802.11a WLAN network is shared by a mix of voice flows encoded with the G.729 standard [32], video flows encoded with the MPEG-4 [33] or the H.263 standards [34], and FTP best effort flows. For the video flows, we have used traffic traces available from the video trace library [35]. For voice flows, we have modeled G.729 sources using Markov ON/OFF sources [36], where the ON period is exponentially distributed with mean value $3 \mathrm{~s}$, and the OFF period has a truncated exponential pdf with an upper limit of $6.9 \mathrm{~s}$ and an average value of 3s [37]. During the ON period, the source sends 20Bytes sized packets every $20 \mathrm{~ms}$ (i.e., the source data rate is $8 \mathrm{kbps}$ ) however, by considering the overheads of the RTP/UDP/IP protocol stacks, the rate becomes $24 \mathrm{kbps}$ during the ON periods. During the OFF period the rate is zero because we assume a Voice Activity Detector (VAD).

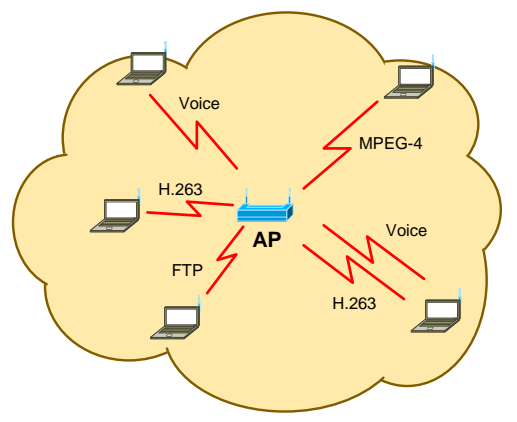

Fig. 4. Scenario with multimedia flows.

We have used the HCCA access method during the CFP, and the EDCA access method during the CP. EDCA parameters have been set as shown in Table 2.

In the $n s-2$ implementation, the $T_{C A}$ is expressed in Time Unit (TU), which in the 802.11 standard [1] is equal to $1024 \mu \mathrm{s}$. We assume a $T_{C A}$ of $29 \mathrm{TU}$. The proportional gain $k_{i}$ is set equal to $1 / \tau_{i}^{T}$. Main characteristics of the considered multimedia flows are summarized in Table 5.

Before starting data transmission, a multimedia source has to set up a new Traffic Stream as specified in Sec. 2.1.2. For that purpose, it sends an ADDTS request and waits for an ADDTS confirm. If this reply is not received within a $\Delta_{T O}$ timeout interval, the admission request is repeated up to a maximum 
Table 5

Main features of the considered multimedia flows.

\begin{tabular}{l|l|l|l|l} 
Type of flow & $\begin{array}{l}\text { Nominal } \\
\text { (Maximum) } \\
\text { MSDU Size }\end{array}$ & $\begin{array}{l}\text { Mean (Max- } \\
\text { imum) Data } \\
\text { Rate }\end{array}$ & $\begin{array}{l}\text { Target } \\
\text { Delay }\end{array}$ & $\begin{array}{l}\text { Inactivity } \\
\text { Interval }\end{array}$ \\
\hline \hline MPEG-4 HQ & $\begin{array}{l}1536(2304) \\
\text { byte }\end{array}$ & $\begin{array}{l}770 \quad(3300) \\
\mathrm{kbps}\end{array}$ & $40 \mathrm{~ms}$ & $3 \mathrm{~s}$ \\
\hline H.263 VBR & $\begin{array}{l}1536(2304) \\
\text { byte }\end{array}$ & $\begin{array}{l}450 \quad(3400) \\
\mathrm{kbps}\end{array}$ & $40 \mathrm{~ms}$ & $3 \mathrm{~s}$ \\
\hline G.729 VAD & $60(60)$ byte & $8.4(24) \mathrm{kbps}$ & $30 \mathrm{~ms}$ & $10 \mathrm{~s}$
\end{tabular}

number of times, $N_{A d m}$ (we have chosen $N_{A d m}=10$ and $\Delta_{T O}=1.5 \mathrm{~s}$ ). If after the $N_{A d m}^{t h}$ request no reply is received back, then the ADDTS request is considered lost and a new admission procedure is initiated after an exponential distributed random time $\Delta_{\text {defer }}$ with average value equal to $1 \mathrm{~min}$.

The duration of video flows is deterministic and equal to $10 \mathrm{~min}$, whereas voice flows durations are exponentially distributed with average value of $2 \mathrm{~min}$. After that a multimedia flows terminates, a new one is generated after an exponentially distributed random time, with average value $1 \mathrm{~min}$. Each terminated flow is withdrawn from the polling list by the $\mathrm{HC}$ after that no more packets from that flow are received for an interval of time equal to the Inactivity Interval reported in Table 5. Each simulation lasts 1hour.

In the sequel, we report some significant comparisons of the proposed dynamic bandwidth allocation and $\mathrm{CAC}$ algorithms with respect to the simple scheduler and the CAC scheme described in the 802.11e draft [21].

We have considered a 802.11a WLAN network shared by a mix of $3 \alpha$ voice flows encoded with the G.729 standard [32], $\alpha$ video flows encoded with the MPEG-4 [33] standard, $\alpha$ video flows encoded with the H.263 standards [34], and $\alpha$ FTP best effort flows. We have tested the proposed algorithm for several values of $\alpha$, herein we report simulation results obtained for $\alpha=5$ and $\alpha=15$. It is worth to point out that, accordingly to data reported in Table 5, and without considering the overhead due to ACK, SIFS, AIFS, for $\alpha=5$ and ( $\alpha=$ $15)$, the average load due to multimedia flows is equal to 6.22 (18.67) Mbps, which is much smaller than the 54Mbps WLAN capacity. On the other hand, the peak load is equal to 33.85 (101.6) Mbps, which is much (smaller) larger than the WLAN capacity. Thus, while with $\alpha=5$ we obtain that the traffic load cannot exceed the WLAN capacity, with $\alpha=15$ we can stress the effects of the burstiness of the multimedia flows.

It should be noted that the CAC scheme proposed in the $802.11 \mathrm{e}$ is static and is entirely based on the average load of the multimedia flows declared in the 
TSPEC. Given that the average load is much smaller than the WLAN capacity (for both $\alpha=5$ and $\alpha=15$ ), we expect a high number of admitted flows, which, due to their bursty behavior, will provoke frequent network overloads, having a negative impact on queuing delays. This effect will be more evident in the case $\alpha=15$, where the peak load is much larger than the WLAN capacity.

Simulation results have confirmed this analysis, in fact, in the case $\alpha=5$ (15), after a 1 hour simulation, 352 (1058) service requests have been sent to the HC, 352 (1027) have passed the CAC test, i.e., 100\% (97\%), whereas (0) 31 requests have been lost due to collision. Regarding delays, Figs. 5 and 6 show the Cumulative Distribution Function (CDF) of the one-way packet delay obtained for $\alpha=5$ and $\alpha=15$, respectively. Fig. 6 shows that the standard bandwidth allocation algorithm cannot provide delay guarantees to multimedia flows in the case $\alpha=15$, since a large fraction of packets have a delay larger than $250 \mathrm{~ms}$. The reason is that a very large number of flows have been admitted and the WLAN capacity is not enough large to serve them. On the other hand, when $\alpha=5$ delays are bounded because the network load due to multimedia flows cannot exceed the WLAN capacity. Finally, for $\alpha=5$ (15) simulations show an average utilization of the superframe for the multimedia flows, which is defined as the sum of the allocated TXOPs over the superframe duration, equal to $26.52 \%(78 \%)$, whereas the peak superframe utilization is equal to $32.42 \%$ (95\%), which means that the network load is very high when $\alpha=15$ and that it is sustainable when $\alpha=5$. It is worth to note that as the load increases the CFP duration becomes larger and, as a consequence, the $\mathrm{CP}$ duration gets smaller. Thus, at high loads, the impact of the bandwidth allocation algorithm becomes crucial since the multimedia flows are served almost exclusively during the CFP.

It should be noted that since the simple scheduler is static, it offers a CBR service, which is not well suited for bursty flows. As a consequence, when the source rate is higher than the average rate, the transmission queue build up and the delay increases. This is the reason for the poor performance shown in Fig. 6.

Now we consider the same scenario with $\alpha=5$ and $\alpha=15$ using the proposed bandwidth allocation along with the CAC algorithm. This time, after a 1 hour simulation, 354 (1987) service requests have been sent to the HC, 354 (698) have passed the CAC test, i. e. 100\%(35\%), whereas, $0(2)$ requests have been lost due to collision. From these results, it is straightforward to note that the proposed CAC scheme has been able to protect the WLAN from heavy overloads. In this case, we have observed an average superframe utilization equal to $6.95 \%(25 \%)$, and a peak utilization equal to $30.52 \%(82 \%)$. This result confirms the bursty nature of multimedia flows so that even though the average load is low, transient overloads cannot be avoided. Figs. 7 and 8 show that in these cases delays are bounded as required by QoS constraints (see Table 5). 


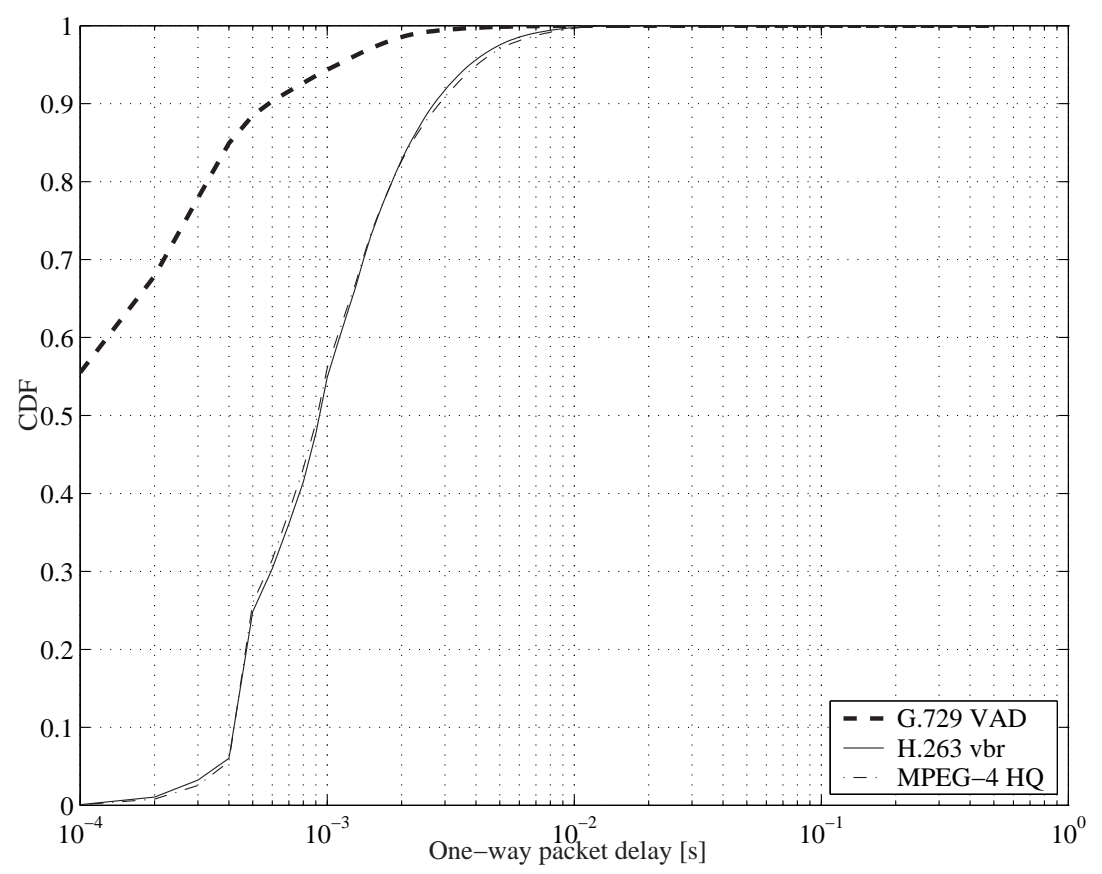

Fig. 5. CDF of the delay obtained using the simple scheduler and the standard CAC when $\alpha=5$.

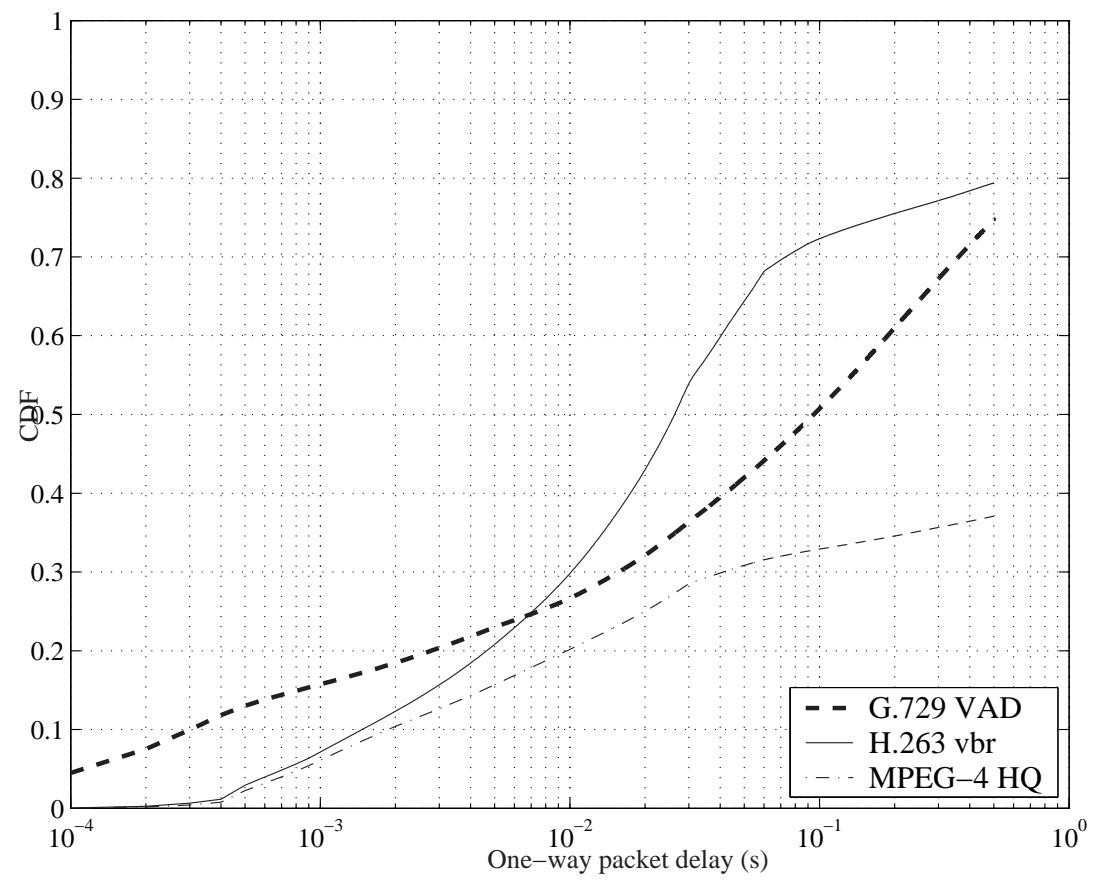

Fig. 6. CDF of the delay obtained using the simple scheduler and the standard CAC when $\alpha=15$.

Finally, in order to give a further insight into the behaviour of the proposed bandwidth allocation algorithm, Figs. 9, 10, and 11 show the bandwidth assigned to a MPEG, a H.263, and a G.729 flow in the case of $\alpha=15$. It is straightforward to note that to effectively serve multimedia flows a dynamic 


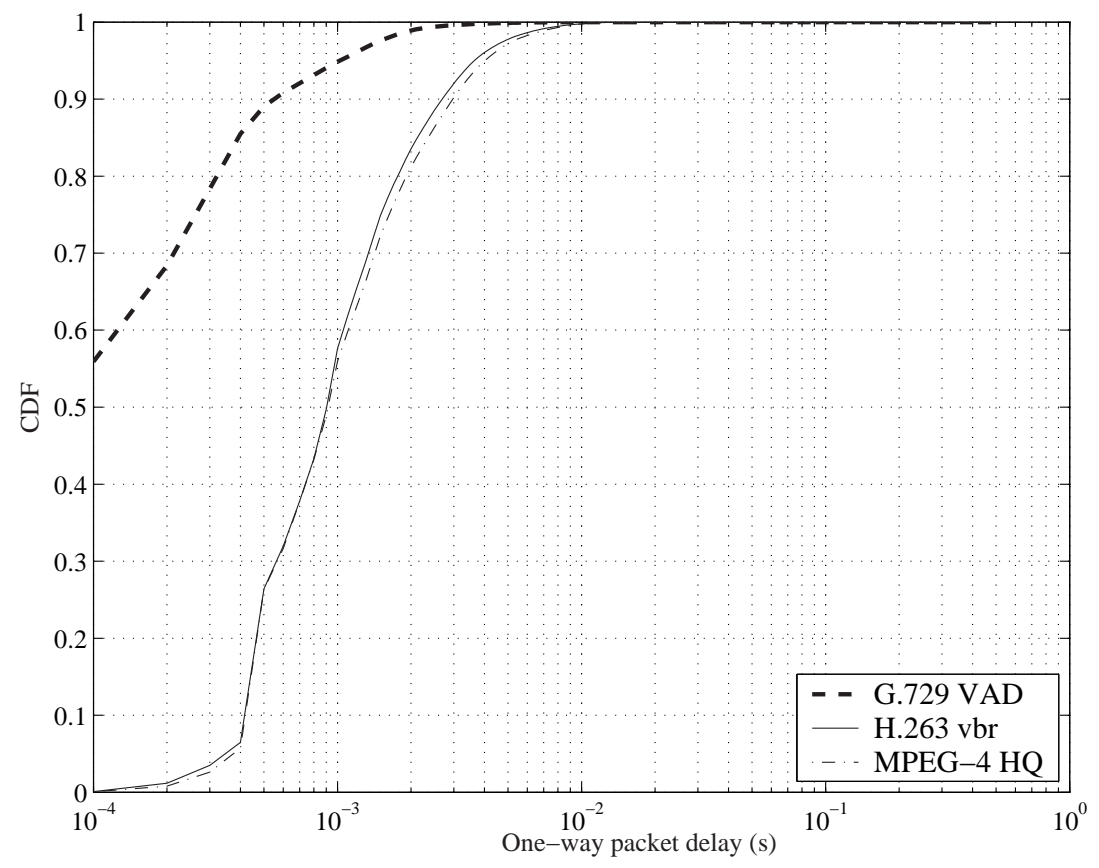

Fig. 7. CDF of the delay obtained using the proposed dynamic bandwidth allocation and $\mathrm{CAC}$ algorithms when $\alpha=5$.

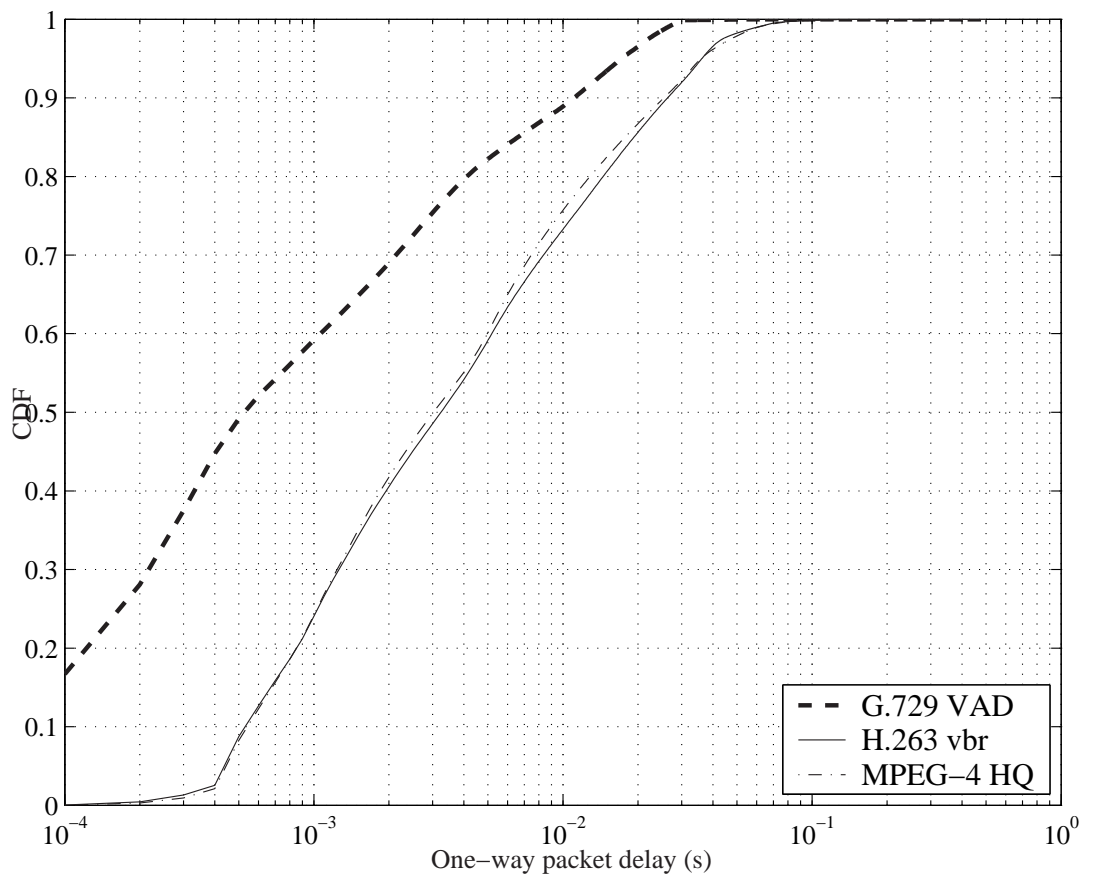

Fig. 8. CDF of the delay obtained using the proposed dynamic bandwidth allocation and CAC algorithms when $\alpha=15$. 
scheduler is required; in fact, the assigned bandwidths exhibit sudden variations in order to track the service requirements of each flow.

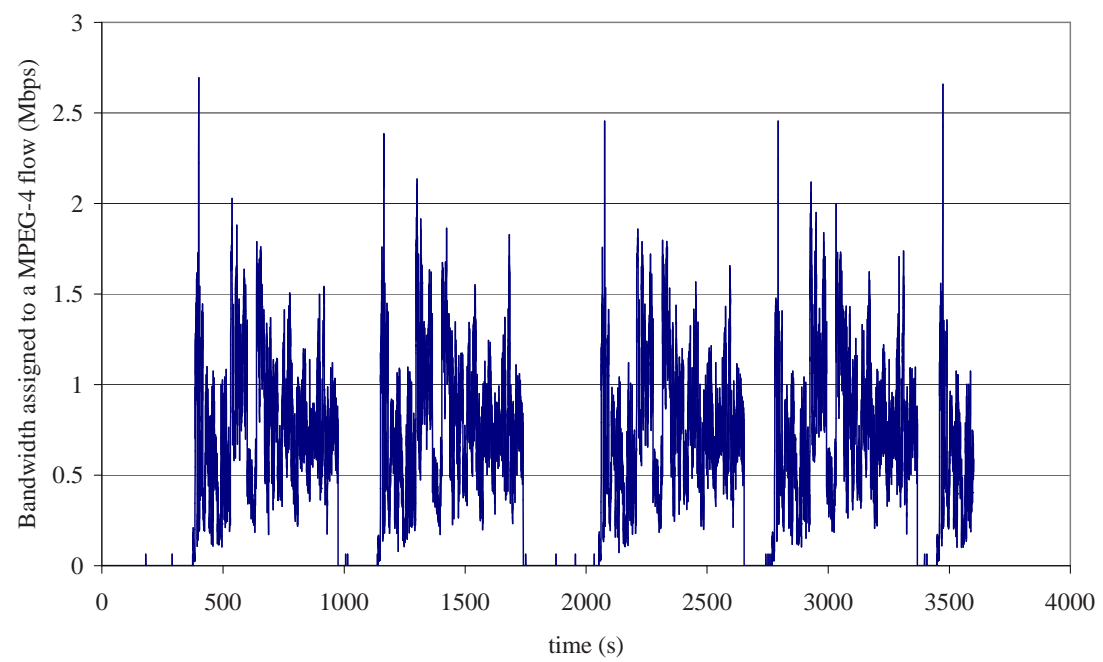

Fig. 9. Bandwidth assigned by the proposed algorithm to a MPEG4 flow when $\alpha=15$.

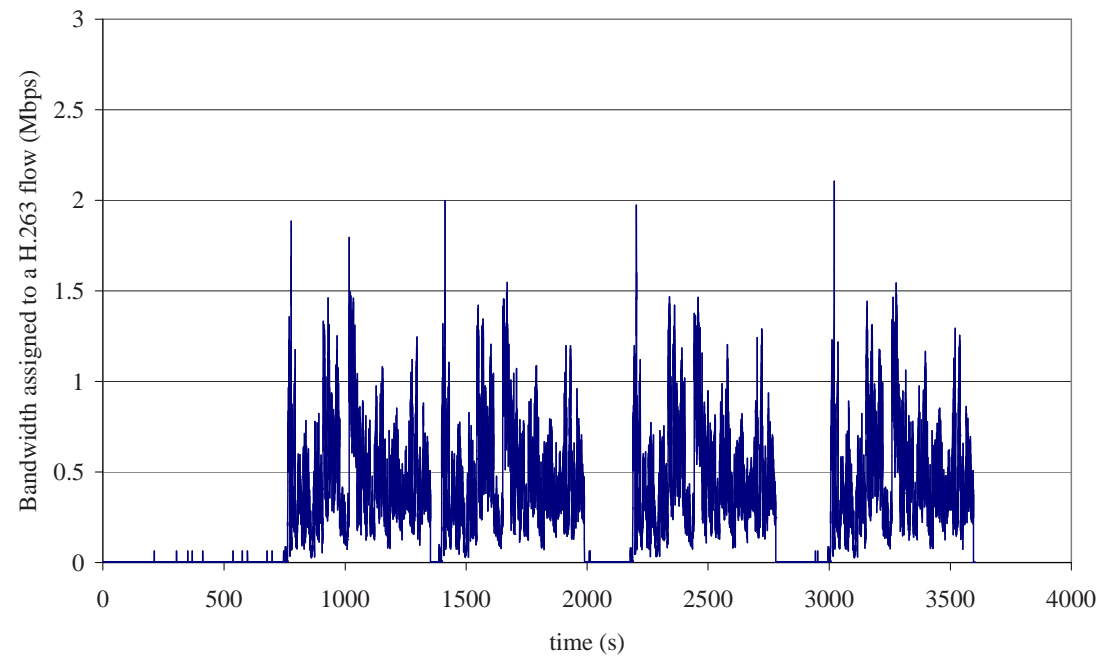

Fig. 10. Bandwidth assigned by the proposed algorithm to a H.263 flow when $\alpha=15$. 


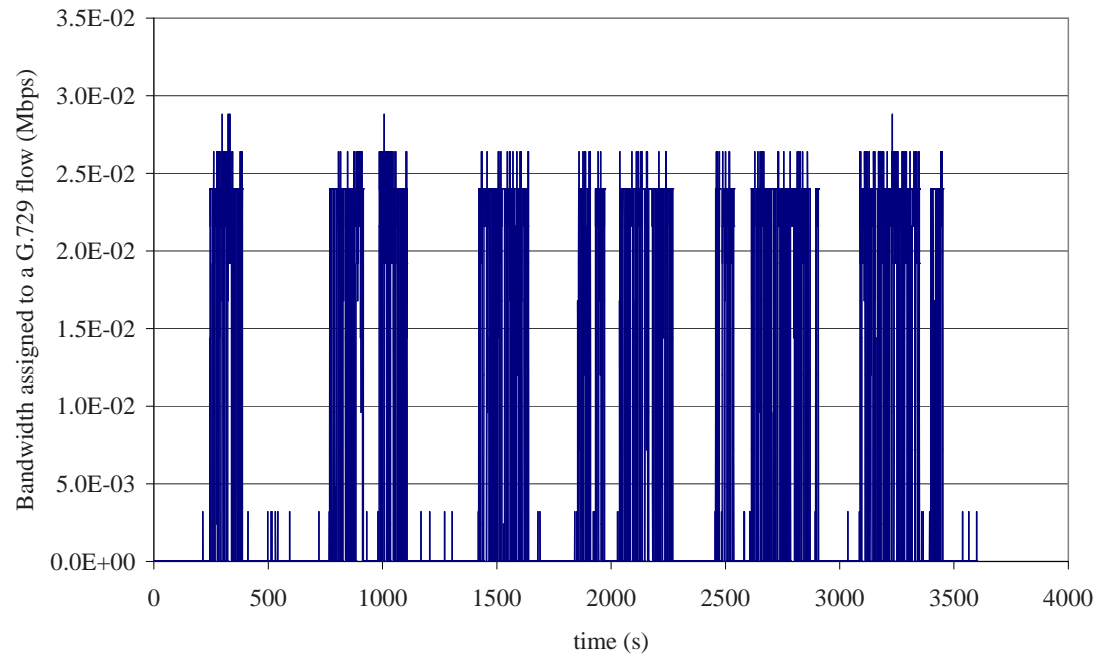

Fig. 11. Bandwidth assigned by the proposed algorithm to a G.729 flow when $\alpha=15$. 


\section{Conclusion and Further Research}

In this paper a dynamic bandwidth allocation algorithm associated with a measurement based CAC algorithm for providing delay guarantees to realtime media flows in IEEE 802.11e networks has been proposed. The dynamic bandwidth allocation algorithm has been designed using classic feedback control theory whereas the CAC scheme is an extension of the one proposed by the 802.11e working group. Both of them exploit the HCF Controlled Channel Access (HCCA) centralized access method.

The performance of the proposed schemes have been compared with the ones obtained using the standard algorithm proposed in the 802.11e draft using the $n s-2$ simulator. Simulation results have shown that the proposed algorithms are able to provide bounded delays to multimedia flows also in high traffic load conditions.

Further research will compare the proposed algorithms with other advanced systems developed for the same problems, such as those proposed in [11] and [12], and will investigate the advantages and drawbacks of more sophisticated control schemes, such as the predictive ones in [38].

\section{References}

[1] IEEE 802.11, Information Technology Telecommunications and Information Exchange between Systems Local and Metropolitan Area Networks Specific Requirements Part 11: Wireless LAN Medium Access Control (MAC) and Physical Layer (PHY) Specifications, ANSI/IEEE Std. 802.11, ISO/IEC 880211, 1st Edition (1999).

[2] U. Varshney, The Status and Future of 802.11-Based WLANs, IEEE Computer 36 (6) (2003) 102-105.

[3] IEEE 802.11, Supplement to IEEE Standard for Information Technology Telecommunications and Information Exchange between Systems Local and Metropolitan Area Networks Specific Requirements Part 11: Wireless LAN Medium Access Control (MAC) and Physical Layer (PHY) Specifications: Higher-Speed Physical Layer Extension in the 5 GHz Band, IEEE Std 802.11a, ISO/IEC 8802-11:1999/Amd 1:2000(E) (1999).

[4] IEEE 802.11, Supplement to IEEE Standard for Telecommunications and Information Exchange Between Systems-LAN/MAN Specific RequirementsPart 11: Wireless LAN Medium Access Control (MAC) and Physical Layer (PHY) Specifications: Further Higher-Speed Physical Layer Extension in the 2.4 GHz Band, IEEE Std 802.11g (2003). 
[5] N. Prasad, A. Prasad (Eds.), WLAN Systems and Wireless IP, Artech House universal personal communications series, Artech House, 2002.

[6] B. P. Crow, I. Widjaja, J. G. Kim, P. T. Sakai, IEEE 802.11 wireless local area networks, IEEE Commun. Mag. (1997) 116-126.

[7] R. LaMaire, A. Krishna, P. Bhagwat, Wireless LANs and mobile networking: Standards and future directions, IEEE Commun. Mag. (1996) 86-94.

[8] S. Mangold, S. Choi, P. May, O. Klein, G. Hiertz, L. Stibor, IEEE 802.11e Wireless LAN for Quality of Service, in: European Wireless Conference 2002, Florence, Italy, 2002.

[9] G. Bianchi, Performance Analysis of the IEEE 802.11 Distributed Coordination Function, IEEE Journal of Selected Areas in Communications 18 (3) (2000) $535-547$.

[10] A. Koepsel, J. P. Ebert, A. Wolisz, A Performance Comparison of Point and Distributed Coordination Function of an IEEE 802.11 WLAN in the Presence of Real-Time Requirements, in: Proc. of 7th Intl. Workshop on Mobile Multimedia Communications (MoMuC2000), Tokio,Japan, 2000.

[11] A. Grilo, M. Macedo, M. Nunes, A scheduling algorithm for QoS support in IEEE 802.11e networks, IEEE Wireless Communications (2003) 36-43.

[12] S. C. Lo, G. Lee, W. T. Chen, An efficient multipolling mechanism for IEEE 802.11 wireless LANs, IEEE Transactions on Computers 52 (6) (2003) 764-778.

[13] K. J. Astrom., B. Wittenmark, Computer controlled systems: theory and design, 3rd Edition, Prentice Hall, Englewood Cliffs, 1995.

[14] S. Mascolo, Congestion control in high-speed communication networks using the smith principle, Automatica, Special Issue on Control methods for communication networks 35 (1999) 1921-1935.

[15] F. D. Priscoli, A. Pietrabissa, Control-theoretic bandwidth-on-demand protocol for satellite networks, in: Proceedings of the 2002 International Conference on Control Applications, 2002, Vol. 1, 2002, pp. $530-535$.

[16] F. D. Priscoli, A. Isidori, A control-engineering approach to traffic control in wireless networks, in: 41st IEEE Conference on Decision and Control (CDC), 2002, Las Vegas, Nevada, USA, 2002, pp. 10-13.

[17] IEEE, Information Technology Telecommunications and Information Exchange between Systems Local and Metropolitan Area Networks Common Specifications Part 3: Media Access Control (MAC) Bridges, ANSI/IEEE Std 802.1D, ISO/IEC 15802-3:1998 (1998).

[18] C. Partridge, A Proposed Flow Specification, IETF RFC 1363 (Sep. 1992).

[19] R. Braden, D. Clark, S. Shenker, Integrated Services in the Internet Architecture: an Overview, IETF RFC 1633 (June 1994). 
[20] S. Blake, D. Black, M. Carlson, E. Davies, Z. Wang, W. Weiss, An Architecture for Differentiated Service, IETF RFC 2475 (Dec. 1998).

[21] IEEE 802.11 WG, Draft Amendment to Standard for Information Technology Telecommunications and Information Exchange between Systems - LAN/MAN Specific Requirements - Part 11: Wireless Medium Access Control (MAC) and Physical Layer (PHY) Specifications: Medium Access Control (MAC) Quality of Service (QoS) Enhancements, IEEE 802.11e/D6.0 (Nov. 2003).

[22] G. Bianchi, I. Tinniriello, Analysis of priority mechanisms based on differentiated inter frame spacing in CSMA-CA, in: IEEE 58th Veichular Technology Conference (VTC03), fall, Orlando, 2003.

[23] D. Gu, J. Zhang, QoS enhancement in IEEE802.11 wireless local area networks, IEEE Commun. Mag. (2003) 120-124.

[24] A. Banchs, X. Perez-Costa, D. Qiao, Providing throughput guarantees in IEEE 802.11e Wireless LANs, in: Providing Quality of Service in Heterogeneous Environments, ITC 2003, Berlin, Germany, 2003, pp. 1001-1010.

[25] P. Garg, R. Doshi, R. Greene, M. Baker, M. Malek, X. Cheng, Using IEEE 802.11e MAC for QoS over Wireless, in: 22nd IEEE International Performance Computing and Communications Conference (IPCCC 2003), Phoenix, Arizona, 2003.

[26] A. Lindgren, A. Almquist, O. Scheln, Quality of service schemes for IEEE 802.11 wireless LANs - an evaluation, Mobile Networks and Applications 8 (3) (2003) $223-235$.

[27] L. Romdhami, Q. Ni, T. Turletti, Adaptive EDCF: Enhanced service differentiation for IEEE 802.11 wireless Ad-Hoc networks, in: IEEE Wireless Communications and Networking Conference (WCNC), New Orleans, Louisiana, USA, 2003.

[28] Y. Xiao, H. Li, S. Choi, Protection and guarantee for voice and video traffic in IEEE 802.11e Wireless LANs, in: IEEE Infocom, Hong Kong, 2004.

[29] G. Boggia, P. Camarda, C. D. Zanni, L. A. Grieco, S. Mascolo, A dynamic bandwidth allocation algorithm for IEEE 802.11e WLANs with HCF access method, in: G. Karlsson, M. I. Smirnov (Eds.), Fourth COST 263 International Workshop on Quality of Future Internet Services (QoFIS 2003), no. 2811 in LNCS, Springer, 2003, pp. 142-151.

[30] International Telecommunication Union (ITU), Transmission Systems and Media, Digital Systems and Networks, ITU-T Recommendation G.1010 (Nov. 2001).

[31] Ns-2, Network simulator, available at http://www-mash.cs.berkeley.edu/ns (2003).

[32] International Telecommunication Union (ITU), Coding of Speech at $8 \mathrm{kbit} / \mathrm{s}$ using Conjugate-Structure Algebraic-Code-Excited Linear Prediction (CSACELP), ITU-T Recommendation G.729 (Mar. 1996). 
[33] MPEG-4 Video Group, Mpeg-4 overview, available at http://mpeg. telecomitalialab.com/ (Mar. 2002).

[34] International Telecommunication Union (ITU), Video coding for low bit rate communication, ITU-T Recommendation H.263 (Feb. 1998).

[35] Video trace library, available at http://www-tkn.ee.tu-berlin.de/research/trace/trace.html.

[36] C. Chuah, R. H. Katz, Characterizing Packet Audio Streams from Internet Multimedia Applications, in: Proc. of International Communications Conference (ICC 2002), New York, NY, 2002.

[37] Y. Xu, R. Guerin, On evaluating loss performance deviation: a simple tool and its pratical implications, in: Quality of Service in multiservice IP networks (QoSIP2003), Milan, Italy, 2003, pp. 1-18.

[38] L. Chisci, R. Fantacci, F. Francioli, T. Pecorella, Multi-terminal dynamic bandwidth allocation in GEO Satellite Networks, in: Proc. of the IEEE Veichular Technology Conference (VTC 2004-Spring), Milan, Italy, 2004. 